\title{
Severe Sepsis and Septic Shock - New Guidelines, 2012
}

\section{Prucha $\mathbf{M}^{*}$}

Department of Clinical Biochemistry, Hematology and Immunology, Na Homolce Hospital, Prague, Czech Republic

Sepsis is the most frequent cause of death at non-coronary intensive care units (ICUs), a serious disease with high mortality and immense financial burden on the health care system. In the past two decades, the incidence of sepsis was on the rise not only in developing countries but also in the USA and countries of Western Europe [1]. In developed countries, the incidence of sepsis is $2 \%$ of all hospitalisations and 6 to $30 \%$ of ICU patients. Even more dramatic is the situation in developing countries [2]. The problem is high mortality of severe sepsis, which is higher than that of myocardial infarction at the moment. Even worse data are revealed in the analysis of septic shock patients [3].

Even though mortality is gradually decreasing, it remains very high at present, about $50 \%$. Unfavourable data have been confirmed by the recent European Sepsis Occurrence in Acutely Ill Patients (SOAP) study, with a $35 \%$ incidence of sepsis in patients at ICUs and mortality of $27 \%$ [4]. In the past 10 years, certain progress was made in early identification of sepsis patients and their treatment. Nevertheless, due to the ever growing incidence of sepsis, the number of patients who die of sepsis increases. The study by Martin et al. from 2009 analysed more than 11,000 patients with severe sepsis from the international register [5]. In this study, $57 \%$ of patients suffered from Gram-negative, $44 \%$ from Gram-positive, and $11 \%$ from mycotic infections. The lungs were the primary source of infection in $47 \%$ of patients, abdominal infection was found in $23 \%$, and urinary tract infection in $8 \%$. The total mortality reached almost $50 \%$.

The Surviving Sepsis Campaign is a collaboration between the US Society of Critical Care Medicine, the European Society of Intensive Care medicine, and the International Sepsis Forum, whose recommendations on the management of sepsis are considered widely. After 4 years, new Surviving Sepsis Recommendations for treatment of severe sepsis and septic shock have been published [6]. What is new in them? If we want to characterise those changes in brief, we can say that some of these recommendations are the outcomes of expert consensus rather than of evidence-based medicine. What are the changes in the management of sepsis? The priority continues to be timely diagnosis of sepsis and active searching in hospitalised patients with a high risk of sepsis development. The objective of diagnostics is finding the focus and targeted surgical intervention along with targeted antibiotic therapy. An approximately $4 \%$ increase in mortality was shown during each hour of delay in the initiation of antibiotic therapy. This increase is cumulative. The empirical antibiotic treatment should not exceed 5 days. In the diagnostics of sepsis, new recommendations are to use mannan and anti-mannan antibody assay and 1,3-beta-D-glucan assay with suspected invasive Candida infection. Regarding other biomarkers, levels of procalcitonin (PCT) higher than two standard deviations (SDs) from the upper limit of the reference range is one of the auxiliary diagnostic parameters. On the contrary, PCT levels $<0.2 \mathrm{ng} / \mathrm{ml}$ is a supporting argument for termination of antibiotic therapy. The concept of 'early goal-directed therapy' has been extended into the Resuscitation Bundles, where the basic treatment measures should be fulfilled under demanding time conditions. Within the first 3 hours it is necessary to measure lactate; within 45 minutes two sets of blood cultures should be performed prior to the initiation of antibiotic therapy and intensive fluid resuscitation should be started, using at least $30 \mathrm{ml} / \mathrm{kg}$ crystalloid; and within 1 hour empirical antibiotic therapy should be initiated.
Immediate initiation of fluid resuscitation before admittance to the ICU has to be emphasised. Within the first 6 hours, it is imperative to achieve a median arterial pressure (MAP) of $65 \mathrm{mmHg}$ using intensive fluid resuscitation and vasopressors. In case of persisting arterial hypotension in spite of fluid resuscitation and/or lactate $\geq 4 \mathrm{mmol} / \mathrm{l}$, the goal is to achieve a central venous pressure (CVP) of $8-12 \mathrm{mmHg}$, superior vena cava oxygen saturation $(\mathrm{ScvO} 2) \geq 70 \%$, or mixed venous oxygen saturation $(\mathrm{SvO} 2 \geq 65 \%$. When using fluid resuscitation, crystalloids are the medicine of choice (1A). The new recommendation is to consider addition of albumin to maintain adequate MAP (2B). The authors strongly recommend against the use of hydroxyethyl starches of molecular weight greater than $200 \mathrm{kDa}$ (1B) because of a more frequent need to use renal replacement therapy (RRT. In case of hypotension, the aim is to achieve a minimum MAP of $65 \mathrm{mmHg}$ using intensive fluid resuscitation. If this is not sufficiently effective, the administration of vasopressors is recommended, the medicine of choice being noradrenaline (norepinephrine) (1B). Adrenaline (epinephrine) remains the second additional agent to maintain MAP (2B). Dopamine is not recommended because of an increased incidence of arrhythmia and higher mortality in some studies. It is reserved for patients with a low risk of arrhythmia incidence, low cardiac output, and low heart rate (2B). The lactate concentration in serum is a marker of global organ hypoperfusion and septic shock. The goal of therapy is normalisation of serum lactate (2B). A normal concentration of lactate, however, is not a reliable indicator of the absence of the state of shock.

It is not recommended to use intravenous immunoglobulins in sepsis treatment. Neither is it recommended to use immunomodulatory supplementation, such as arginine, glutamine, omega 3 , or antioxidants. The administration of systemic corticosteroids is reserved for those patients with septic shock in which normalisation of hypotension fails using fluid resuscitation and vasopressors. Continuing administration of hydrocortisone at a dose of $200 \mathrm{mg} /$ day is given preference.

The most important principles of the new guidelines can be briefly summed up as follows: early recognition, diagnostics, and early treatment.

\section{References}

1. Martin GS (2012) Sepsis, severe sepsis and septic shock: changes in incidence, pathogens and outcomes. Expert Rev Anti Infect Ther 10: 701-706.

2. Jawad I, Lukšić I, Rafnsson SB (2012) Assessing available information on the burden of sepsis: global estimates of incidence, prevalence and mortality. $J$ Glob Health 2: 10404.

*Corresponding author: Miroslav Prucha, Department of Clinical Immunology, Na Homolce Hospital, Prague 5, Czech Republic, Tel: +420 257272433; +420 257273150; E-mail: miroslav.prucha@homolka.cz

Received November 27, 2013; Accepted November 28, 2013; Published December 07, 2013

Citation: Prucha M (2013) Severe Sepsis and Septic Shock - New Guidelines, 2012. Clin Microbial 3: e117. doi:10.4172/2327-5073.1000e117

Copyright: () 2013 Prucha M. This is an open-access article distributed under the terms of the Creative Commons Attribution License, which permits unrestricted use, distribution, and reproduction in any medium, provided the original author and source are credited. 
3. Esper A, Martin GS (2007) Is severe sepsis increasing in incidence AND severity? Crit Care Med 35: 1414-1415.

4. Vincent JL, Sakr Y, Sprung CL, Ranieri VM, Reinhart K, et al. (2006) Sepsis in European intensive care units: results of the SOAP study. Crit Care Med 34 344-353.
5. Martin G, Brunkhorst FM, Janes JM, Reinhart K, Sundin DP, et al. (2009) The international PROGRESS registry of patients with severe sepsis: drotrecogin alfa (activated) use and patient outcomes. Crit Care 13: R103.

6. Dellinger RP, Levy MM, Rhodes A, Annane D, Gerlach H, et al. (2013) Surviving Sepsis Campaign: international guidelines for management of severe sepsis and septic shock, 2012. Intensive Care Med 39: 165-228. 